\title{
Endovascular Repair of Iliac Artery Aneurysms: A Single Center Experience in 10-Years
}

\author{
Onur S. Goksel, MD, ${ }^{1}$ Emre Gok, MD,${ }^{1}$ Akif Onalan, MD, ${ }^{2}$ Koray Guven, MD,${ }^{3}$ Gazi Çapar, MD, ${ }^{1}$ \\ Bayer Cinar, MD, ${ }^{4}$ Ufuk Alpagut, $\mathrm{MD}^{1}$ \\ ${ }^{1}$ Cardiovascular Surgery Department, Istanbul Medical Faculty, Istanbul University, Istanbul, Turkey; ${ }^{2}$ Cardiovascular Surgery \\ Department, Mehmet Akif Inan Education And Research Hospital, Sanliurfa, Turkey; ${ }^{3}$ Radiology Department, Faculty of Medicine, \\ Acibadem Mehmet Ali Aydinlar University, Istanbul, Turkey; ${ }^{4}$ Cardiovascular Surgery Department, Altunba University, Istanbul, Turkey
}

\section{ABSTRACT}

Objectives: Isolated iliac artery aneurysms (IAAs) are rare, but nonetheless life-threatening when ruptured. The endovascular approach has taken over open repairs in time. The reported data is constituted of a retrospective series. We reviewed our 10-year-long experience with elective endovascular treatment of iliac aneurysms.

Methods: Data regarding 22 patients with 24 IAAs treated with endovascular stent grafting between 2005 and 2015 were reviewed.

Results: Twenty-two patients (aged $68.4 \pm 9.6$ years, range 50-82) with 24 unilateral or bilateral iliac aneurysms were treated. Twenty patients (91\%) were male. Two patients with unilateral IAA had prior abdominal aortic aneurysm (AAA) surgical repair. The mean aneurysm diameter was $4.8 \pm 2.1$ (3.8 to 7.1 ) $\mathrm{mm}$. Procedural success rate was $100 \%$; only one patient with an iliovenous fistula had periprocedural type II endoleak. Internal iliac artery coil occlusion was applied in 16 of 24 procedures $(66 \%)$. Thirty-day mortality included one patient (4\%).

Conclusion: Endovascular repair is the preferred approach for isolated IAAs. Because of the retrospective nature of data sets, larger cohorts are necessary for better definition of morbidity and mortality rates.

\section{INTRODUCTION}

Iliac artery aneurysms (IAAs) are uncommon and are frequently associated with coexisting abdominal aortic aneurysms (AAAs). Isolated IAAs are even less frequent, accounting for less than $2 \%$ of all intra-abdominal aneurysms [Richardson 1988; Levi 1998]. Most IAAs are asymptomatic;

Received March 14, 2018; accepted October 2, 2018.

Abbreviations: AAA, abdominal aortic aneurysm; CTA, computed tomography angiography; EVIR, endovascular repair of iliac artery aneurysms; EVAR, endovascular repair of abdominal aortic aneurysm; IAA, iliac artery aneurysm.

Correspondence: Dr. Onur S. Goksel, Istanbul Medical Faculty, Millet Cd. ÇAPA, Istanbul, Turkey; +90-212-414-20-00; fax: +90-216-348-02-69 (e-mail: onurgokseljet@gmail.com). patients may, however, present with rupture, distal embolization, thrombosis, and symptoms of visceral or neurologic compression. Similar to AAAs, IAAs are prone to rupture when they reach a critical size; unfortunately, data on the natural history of untreated IAAs are scarce. McCready et al observed a growth rate of $4 \mathrm{~mm} /$ year and recommended repair when they reached $3 \mathrm{~cm}$ [McCready 1983]. Santilli et al studied the rate of growth of isolated IAAs, the most common IAAs. IAAs $<3 \mathrm{~cm}$ expanded at an average rate of $1.1 \mathrm{~mm} /$ year, whereas those $>3 \mathrm{~cm}$ expanded by $2.6 \mathrm{~mm} /$ year [Santilli 2000]. Because symptoms, including rupture, did not develop with IAAs $<4 \mathrm{~cm}$ in diameter, the authors suggested repair for aneurysms $>3.5 \mathrm{~cm}$. The operative mortality of ruptured IAAs may be as high as $40 \%$ even with contemporary anesthetic and surgical techniques. IAA-associated mortality is thus mostly prevented by identifying patients at risk of rupture and prophylactic repair [Brunkwall 1989; Krupski 1998]. Furthermore, elective repair of IAA is often technically challenging given the pelvic location and the frequent history of prior abdominopelvic surgery including AAA repair. This may account for the higher mortality associated with elective common iliac and hypogastric aneurysm repairs compared to AAA repair [Katz 1994; Krupski 1998].

Over the past decade, endovascular aortoiliac aneurysm repair has been established as a safe and effective alternative to open repair in patients with appropriate anatomy. We

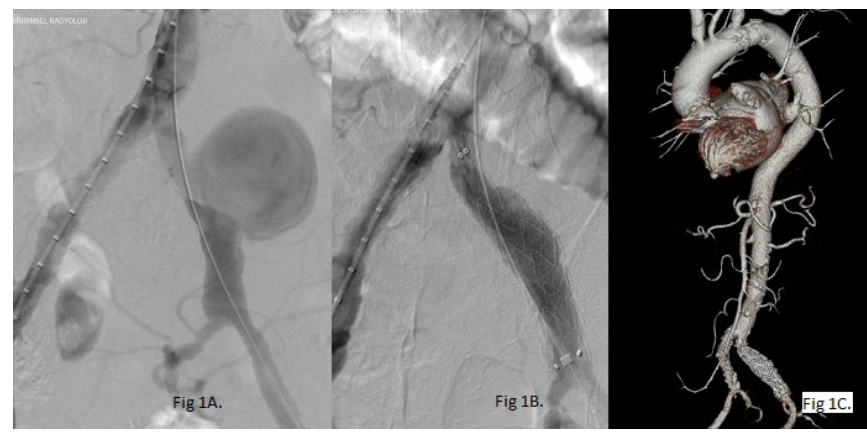

Figure 1. Preoperative digital subtraction angiogram (DSA) of an isolated common IAA (A). Immediate postoperative DSA showing successful exclusion of the aneurysm (B). Postoperative 12th-month CT angiogram of the same patient without any complications (C). 


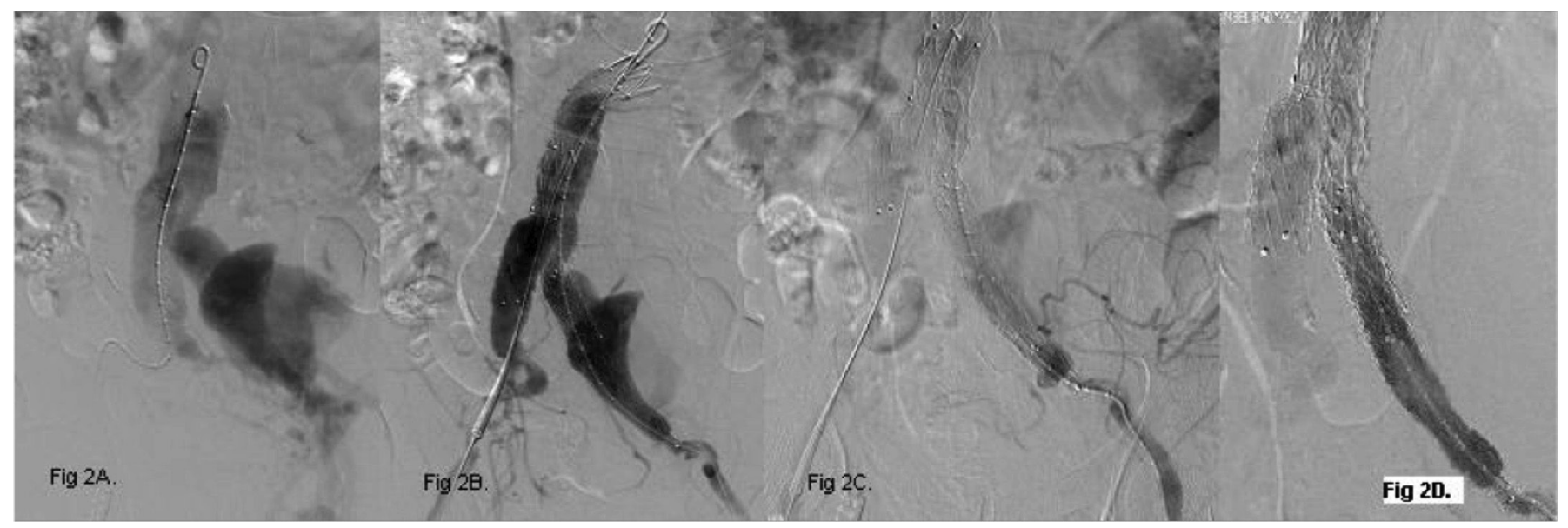

Figure 2. Preoperative digital subtraction angiogram (DSA) of an isolated common IAA with fistulation into the inferior vena cava (A). Exclusion of the aneurysm and the fistula with an aortobi-iliac endograft with continuing contrast filling on the venous side (B). Selective angiogram of the side branch filling the aneurysmal sac (C). Successful clip ligation of the particular side branch and the discontinued contrast filling of the sac and the caval side (D).
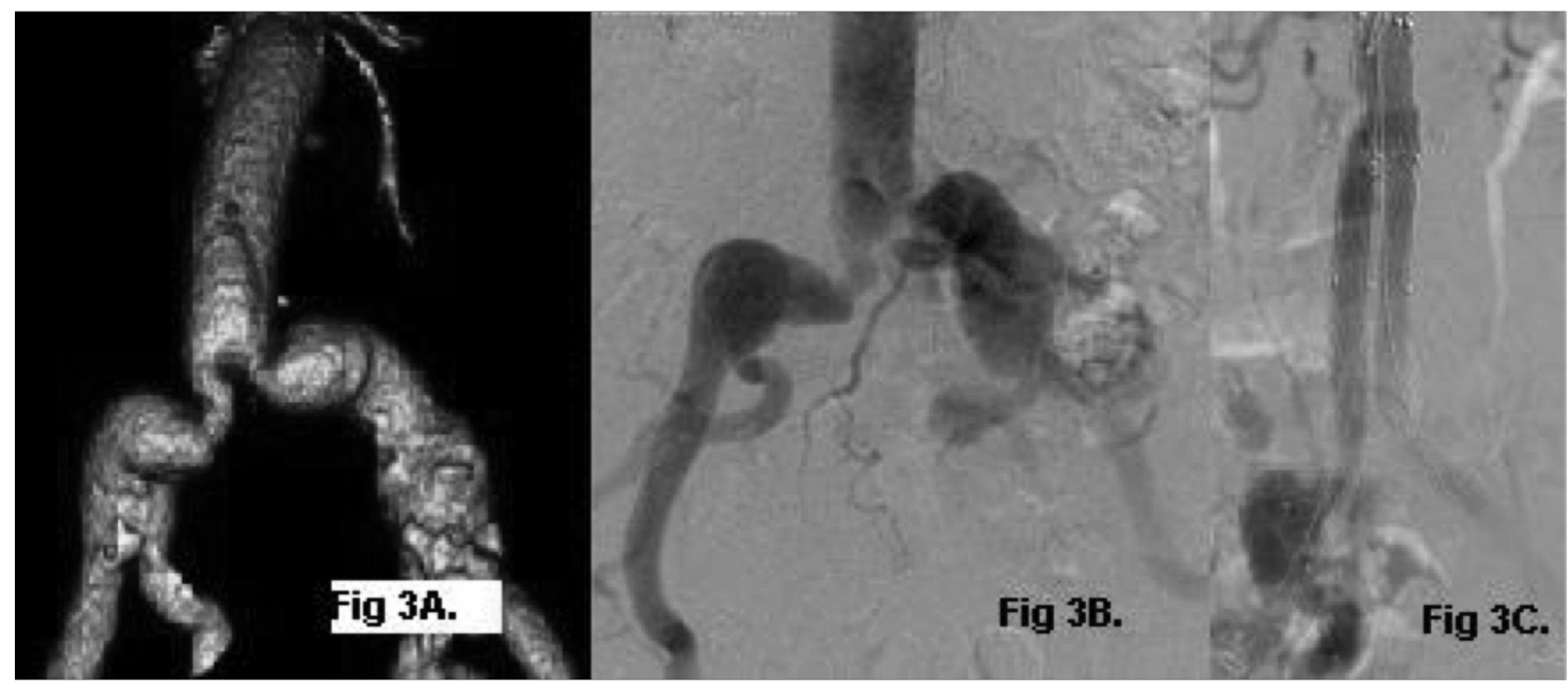

Figure 3. Preoperative CAT scan (A) and digital subtraction angiogram (DSA) (B) of a patient with a prior tube graft repair for AAA. Post-EVAR DSA of the patient (C).

have reviewed our experience with endovascular repair of iliac artery aneurysms (EVIR) of isolated IAAs.

\section{PATIENTS AND METHODS}

Twenty-two patients (aged $68.4 \pm 9.6$ years, range $50-78$ ) referred to our clinic with an aneurysm involving common iliac arteries between 2005 and 2015 were reviewed. Patients with IAAs larger than $4 \mathrm{~cm}$ in diameter and those with common IAAs involving aortic bifurcation were included. Patients with isolated internal IAAs or those with aortoiliac aneurysms were excluded. Preoperative radiographic evaluation consisted of computed tomography angiography (CTA) in all cases, which was used to size the aneurysm and endograft.
Hospital records and early and midterm follow-ups were reviewed upon Institutional Scientific Committee approval from each author's institution. All patients had control CTA scans at the third month and then annually if asymptomatic. Medical status including cardiovascular signs upon examination was prospectively recorded at each control visit. All survivors conformed to the follow-up protocol. All patients were operated on through the femoral route with femoral cutdown under local anesthesia unless his/her hemodynamic status became compromised.

Endovascular repair has become the preferred approach at our institution for patients presenting with an isolated IAA. Patients treated via an endovascular approach underwent unilateral iliac endograft placement if there was an 
Postoperative Outcome of Contemporary EVIR for IAAs

\begin{tabular}{|c|c|c|c|c|c|}
\hline Number of patients & 22 & 32 & 32 & 45 & 52 \\
\hline Age, years $[$ mean $\pm S D]$ & $68.4 \pm 9.6$ & $74 \pm 10$ & $64 \pm 8$ & $75 \pm 9$ & $73 \pm 2$ \\
\hline Gender, male [patient nos. (\%)] & $20(91 \%)$ & $31(97 \%)$ & $30(94 \%)$ & $42(93 \%)$ & $47(90 \%)$ \\
\hline Technical success & $100 \%$ & $100 \%$ & $100 \%$ & $100 \%$ & $100 \%$ \\
\hline Need for transfusion [patient nos. (\%)] & $1 / 22(4.5 \%)$ & $1 / 32(3.1 \%)$ & 0 & - & $3 / 52(6 \%)$ \\
\hline 30-Day morbidity [patient nos. (\%)] & $1 / 22(4.5 \%)$ & $6 / 32(19 \%)$ & $6 / 32(19 \%)$ & $2 / 45(4 \%)$ & $8 / 52(15 \%)$ \\
\hline 30-Day mortality [patient nos. (\%)] & $1 / 22(4.5 \%)$ & 0 & 0 & 0 & $1 / 52(2 \%)$ \\
\hline Primary patency, 24 months & - & $96 \%$ & $97 \%$ & $95 \%$ & $81 \%$ \\
\hline
\end{tabular}

*Goksel et al refers to this article. The others refer to the corresponding items in the reference list.

†Series including isolated IAAs.

appropriate neck (usually $10 \mathrm{~mm}$ ) present in the proximal common iliac artery. Otherwise, a bifurcated aortic endograft was placed. In all instances, the endograft limb was placed into the external iliac artery landing zone (usually $>20 \mathrm{~mm}$ ). The ipsilateral internal iliac artery was addressed by covering the ostium with an endograft with or without coil embolization (Cook Medical, Bloomington, IN, USA). Coil embolization was performed prior to endograft placement, either as a separate procedure or at the same time. Embolization coils were restricted to the main internal iliac artery trunk if only a common IAA was treated. Coils were placed in the anterior and posterior divisions and within the main trunk if an internal IAA was treated.

\section{RESULTS}

Between 2005 and 2015, 22 patients (aged $68.4 \pm 9.6$ years, range 50-82) with 24 unilateral or bilateral iliac aneurysms were treated. EVIR was performed in all patients with local anesthesia except in an octogenarian patient in whom an emergency endotracheal intubation was required because of cardiopulmonary arrest during the procedure. Twenty patients $(91 \%)$ were male. All patients underwent EVIR in elective settings besides the patient with an iliovenous fistula. The mean aneurysm diameter was $4.8 \pm 2.1$ (3.8 to 7.1$) \mathrm{mm}$. Among the total of 24 IAAs, 4 patients including the patient with de novo bilateral IAAs received aortobi-iliac endografts, and 22 aorto-uni-iliac endografts (Figure 1) were employed. Procedural success rate was $100 \%$; only one patient with an iliovenous fistula had periprocedural type II endoleak (Figure 2). Internal iliac artery coil occlusion was applied in 16 of 24 procedures (66\%). Thirty-day mortality included one patient who necessitated cardiopulmonary resuscitation and had multiorgan failure in the postoperative period; he unfortunately died on day 17 (4\%). Blood transfusion rate was minimal in the group and was necessary in only the patient described above. Procedure-related morbidity included only one patient with a transient femoral lymphorrhea (3 days). First year follow-up was complete in all patients besides the one patient who died. Significant sac diameter decrease $(>5 \mathrm{~mm})$ within one year was observed in 7 patients. At the end of one year, 2 patients had sac enlargement $(2$ and $3 \mathrm{~mm}$, respectively) due to type $\mathrm{Ib}$ endoleak in one patient and no detectable etiology in the other. Limb extension was performed in the former patient at the 12 th-month control visit. The sac diameter, however, remained stable in 10 patients.

It is noteworthy that the patient with an iliovenous fistula remained stable without sac enlargement at the end of the 1st year despite the multiple interventions to eliminate type II endoleaks caused by the extensive network between the arteries and veins of the posterior abdominal wall. Surgical ligation of the side branches served as a temporary solution and a type II endoleak returned after the third month (Figures 2C and 2D). Two patients with unilateral IAA had a history of open AAA repair (one patient with an aortic tube graft, the other with an aortobi-iliac bifurcation graft), and 4 patients had endovascular repair of abdominal aortic aneurysm (EVAR) for AAA. One of the latter patients had bilateral IAAs (Figure 3).

\section{DISCUSSION}

Isolated IAAs are uncommon, with an incidence less than $2 \%$ of intra-abdominal aneurysms [Richardson 1988; Levi 1998]. The primary etiology is degenerative; however, isolated IAAs are also caused by other predisposing conditions, 
including infection, intraoperative injury, dissection, trauma, Marfan syndrome, and other collagen vascular diseases [Patel 2009]. Over the past decade, endovascular aortoiliac aneurysm repair has been established as a safe and effective alternative to open repair in patients with appropriate anatomy. Pelvic anatomic and technical considerations that increase the potential for morbidity and mortality during open repair of isolated IAA make endovascular repair particularly attractive. Avoidance of the need for deep pelvic dissection, with the attendant risks of visceral, genitourinary, and pelvic venous injuries, is the putative benefit of the endovascular approach; therefore, comparison with a control group (ie, surgical group) is not possible for the surgeons' particular preference.

This series includes 22 consecutive patients treated with elective EVIR for isolated degenerative IAAs. Our 100\% technical success with low morbidity and mortality rates is concordant with the several series demonstrating morbidity rates as low as $4 \%$ to $15 \%$ with a corresponding decrease in transfusion with endovascular repair of isolated IAAs [Boules 2006; Pitoulias 2007; Chaer 2008] Except for the one patient who died, no patients required transfusion as in other contemporary series (Table). Endoleak rates are also comparable. Unfortunately, patency rates were not specifically looked at for the study; however, limb ischemia was not observed in the series; thus. an additional limb-salvage procedure (percutaneous or surgical) was not applied in any of the patients in contrast to the above-mentioned series. It is noteworthy that our series include only 12-month-long follow-up in contrast to 24-month-long follow-ups reported [Boules 2006; Pitoulias 2007; Chaer 2008; Patel 2009].

At the end of one year, significant sac diameter decrease ( $5 \mathrm{~mm}$ or more/year) was observed in 7 patients in addition to 10 patients in whom the sac diameter remained stable. Of note, 2 patients had sac enlargement ( 2 and $3 \mathrm{~mm}$, respectively) due to type Ib endoleak in one patient and no detectable etiology in the other. As stated before, a limb extension was performed in the former patient. This finding is also comparable to other major series where sac diameter remained stable or even decreased [Pitoulias 2007; Huang 2008; Patel 2009].

Similar to the trend seen in AAA repair, treatment of isolated IAA has shifted away from open surgical repair toward a less invasive endovascular technique [Giles 2009; Buck 2015]. The total number of isolated IAA repairs has increased with the introduction of EVIR, likely in part because of increased detection related to increased use of abdominal imaging over time [Giles 2009]. However, unlike what was seen with AAA, the increase in elective procedures may not have led to a decrease in urgent procedures over time. This suggests that the increase in elective procedures may be primarily related to a lower threshold for intervention with the availability of a less invasive treatment. The decrease in isolated IAA inhospital deaths over time is likely related to the reduction in elective mortality, similar to trends observed in AAA treatment [Buck 2015].

The main limitation of this study is that it is a retrospective review with limited sample size and only one-year followup of this cohort. A prospective algorithm for treatment and follow-up was not used; technical and treatment decisions were made on the basis of the best clinical judgment by the primary surgeon. Considerations for endovascular repair included adequacy of the access vessel and proximal attachment site, and method for addressing the ipsilateral internal iliac artery. It is possible that true differences exist between certain variables, but conclusions are hindered by relatively smaller series. Similarly, the current experience only reflects the elective cases; the outcome is to be sought with the patients in emergent situations where the periprocedural morbidity and mortality are likely to differ.

Nonetheless, our results add to a growing body of data reported worldwide suggesting that EVIR is an effective and safe alternative to open repair for this rare pathology in anatomically appropriate patients.

\section{REFERENCES}

Boules TN, Selzer F, Stanziale SF, et al. 2006. Endovascular management of isolated iliac artery aneurysms. J Vasc Surg 44:29-37.

Brunkwall J, Hauksson H, Bengtsson H, Bergqvist D, Takolander R, Bergentz SE. 1989. Solitary aneurysms of the iliac arterial system: an estimate of their frequency of occurrence. J Vasc Surg 10:381-4.

Buck DB, Bensley RP, Darling J, et al. 2015. The effect of endovascular treatment on isolated iliac artery aneurysm treatment and mortality. J Vasc Surg 62:331-5.

Chaer RA, Barbato JE, Lin SC, Zenati M, Kent KC, McKinsey JF. 2008. Isolated iliac artery aneurysms: a contemporary comparison of endovascular and open repair. J Vasc Surg 47:708-13.

Giles KA, Pomposelli F, Hamdan A, Wyers M, Jhaveri A, Schermerhorn ML. 2009. Decrease in total aneurysm-related deaths in the era of endovascular aneurysm repair. J Vasc Surg 49:543-50; discussion and invited commentary 550-1.

Huang Y, Gloviczki P, Duncan AA, et al. 2008. Common iliac artery aneurysm: expansion rate and results of open surgical and endovascular repair. J Vasc Surg 47:1203-1211.e2.

Katz DJ, Stanley JC, Zelenock GB. 1994. Operative mortality rates for intact and ruptured abdominal aortic aneurysms in Michigan: an elevenyear statewide experience. J Vasc Surg 19:804-15; discussion 816-7.

Krupski WC, Selzman CH, Floridia R, Strecker PK, Nehler MR, Whitehill TA. 1998. Contemporary management of isolated iliac aneurysms. J Vasc Surg 28:1-11; discussion 11-3.

Levi N, Schroeder TV. 1998. Isolated iliac artery aneurysms. Eur J Vasc Endovasc Surg 16:342-4.

McCready RA, Pairolero PC, Gilmore JC, Kazimer FJ, Cherry KJ Jr, Hollier LH. 1983. Isolated iliac artery aneurysms. Surgery 93:688-93.

Patel NV, Long GW, Cheema ZF, Rimar K, Brown OW, Shanley CJ. 2009. Open vs. endovascular repair of isolated iliac artery aneurysms: a 12-year experience. J Vasc Surg 49:1147-53.

Pitoulias GA, Donas KP, Schulte S, Horsch S, Papadimitriou DK. 2007. Isolated iliac artery aneurysms: endovascular versus open elective repair. J Vasc Surg 46:648-54.

Richardson JW, Greenfield LJ. 1988. Natural history and management of iliac aneurysms. J Vasc Surg 8:165-71.

Santilli SM, Wernsing SE, Lee ES. 2000. Expansion rates and outcomes for iliac artery aneurysms. J Vasc Surg 31(1 Pt 1):114-21. 